\title{
Placebo ebenso wirksam wie die Behandlung mit Amitriptylin oder Topiramat
}

Fragestellung: Reduzieren Amitriptylin oder Topiramat die Kopfschmerzfrequenz und die alltäglichen Einschränkungen bei der Prophylaxe der kindlichen Migräne?

Hintergrund: Etwa $5 \%$ aller Kinder und Jugendlichen leiden an Migräne. Diese geht mit einer signifikanten Einschränkung der Lebensqualität und einer hohen gesundheitsökonomischen Belastung einher. Therapieprinzipien basieren überwiegend auf Konsensusempfehlungen. Eine für Kinder unter zwölf Jahren zugelassene Arzneimitteltherapie fehlt bisher.

Patienten und Methodik: In einer randomisierten, doppelblinden, placebokontrollierten Phase-III-Studie wurden 361 Patienten im Alter zwischen acht und 17 Jahren mit Migräne mit und ohne Aura in den USA untersucht. Die Patienten wiesen eine moderate bis schwere alltägliche Einschränkung sowie vier oder mehr Kopfschmerztage innerhalb der letzten 28 Tage auf. Nach einer Baseline-Phase von 28 Tagen erfolgte die Randomisierung zu Amitriptylin-, Topiramat- und Placebogruppe im Verhältnis 2:2:1 und die 24-wöchige Testphase. Die Arzneimitteldosis war gewichtsadaptiert (Amitriptylin $1 \mathrm{mg} / \mathrm{kg}$ Körpergewicht [KG], Topiramat $2 \mathrm{mg} / \mathrm{kg} \mathrm{KG}$ ); es folgte eine Dosissteigerung unter Berücksichtigung unerwünschter Arzneimittelwirkungen. Der primäre Endpunkt war eine relative Reduktion der Anzahl an Kopfschmerztagen im Ver-

Powers SW, Coffey CS, Chamberlin LA et al. Trial of amitriptyline, topiramate and placebo for pediatric migraine. $N$ Engl J Med 2017; 376: $115-24$ gleich zur 28-tägigen Baseline-Phase um $\geq 50 \%$. Sekundäre Endpunkte waren die alltägliche Einschränkung, die absolute Reduktion der Kopfschmerztage, die
Anzahl der versuchsabschließenden Patienten sowie schwerwiegende unerwünschte Ereignisse.

Ergebnisse: Die Studie wurde aufgrund fehlenden Nutzens vorzeitig beendet. Die Studienpopulation unterschied sich in den Basischarakteristika nicht signifikant. Die mittlere Dosis betrug $0,99 \pm 0,18 \mathrm{mg} / \mathrm{kg} \mathrm{KG}$ für Amitriptylin und 1,93 $\pm 0,40 \mathrm{mg} / \mathrm{kg}$ KG für Topiramat. Den primären Endpunkt erreichten $52 \%$ der Amitriptylin- (im Vergleich zu Placebo p = 0,26), $55 \%$ der Topiramat- (im Vergleich zu Placebo $\mathrm{p}=0,48)$ und $61 \%$ der Placebogruppe. Hinsichtlich der sekundären Endpunktes ergab sich kein signifikanter Gruppenunterschied in der alltäglichen Beeinträchtigung, der absoluten Kopfschmerztage und der Abbruchrate der Studie. Unerwünschte Wirkungen waren insgesamt signifikant häufiger in den Verumgruppen, mit unterschiedlicher Verteilung für einzelne Nebenwirkungen. Signifikant häufiger in der Amitriptylin-Gruppe (im Vergleich zu Placebo) traten Müdigkeit und Mundtrockenheit auf, in der Topiramat-Gruppe (im Vergleich zu Placebo) Parästhesien und Gewichtsverlust. Es traten fünf therapieassoziierte schwerwiegende unerwünschte Ereignisse auf: drei Ereignisse einer alterierten Stimmung und eine Synkope in der Amitriptylin-Gruppe sowie ein Suizidversuch in der Topiramat-Gruppe.

Schlussfolgerungen: Weder Amitriptylin noch Topiramat waren in der Prophylaxe der kindlichen Migräne hinsichtlich des primären Endpunkts wirkungsvoller als Placebo. Unerwünschte Ereignisse waren unter Amitriptylin und Topiramat signifikant häufiger als in der Placebogruppe. Der deutliche Placeboeffekt (ähnlich wie in vorherigen Studien) könnte zukünftig möglicherweise vorteilhaft eingesetzt werden.

\section{- Kommentar von Ulrike Bingel und Julian Kleine-Borgmann, Essen}

\section{Weitere kontrollierte Studien sind dringend notwendig}

Das Ergebnis der Studie unterstützt die gängigen Leitlinienempfehlungen, vorrangig nicht medikamentöse Strategien zur Behandlung der Migräne im Kindes- und Jugendalter einzusetzen. Leider war das Randomisierungsverhältnis von Verum zu Placebo von 4:1 sehr ungünstig gewählt. Die hohe Wahrscheinlichkeit - und damit Erwartung - das Verum zu erhalten, erhöht die Placeborate und verringert die Assay-Sensitivität für den Therapieeffekt des Verums. Auch waren die gewählten Dosierungen deutlich höher als üblicherweise im klinischen Alltag eingesetzt, was die nicht unerhebliche Rate an Nebenwirkungen erklärt. Es bleibt zu hoffen, dass in Zukunft weitere kontrollierte Studien zur Behandlung der Migräne im Kindesund Jugendalter durchgeführt werden, dann hoffentlich mit optimalerem Design. Diese sind dringend notwendig, um eine bessere Datengrundlage für die Therapie der Kinder und Jugendlichen zu bekommen, bei denen eine nicht medikamentöse Behandlung der Migräne eben nicht ausreicht.

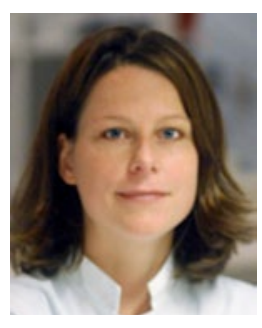

Prof. Dr. med. Ulrike Bingel, Essen

Lehrstuhl für funktionelle Bildgebung, Leitung der Schmerzambulanz, Klinik für Neurologie der Universitätsklinik Essen E-Mail: ulrike.bingel@uk-essen.de 